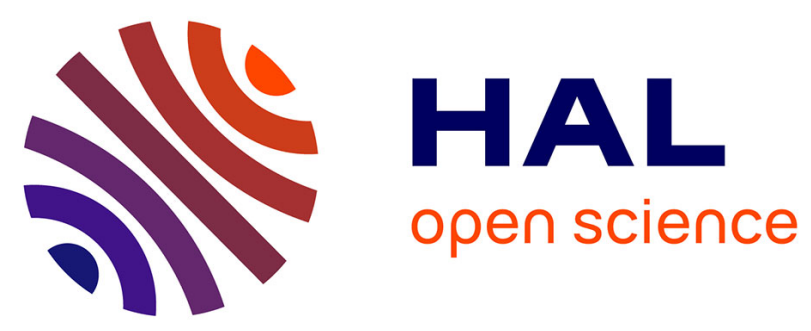

\title{
Static pressure recovery analysis in the vane island diffuser of a centrifugal pump
}

Qiaorui Si, Patrick Dupont, Annie-Claude Bayeul-Lainé, Antoine Dazin, Olivier Roussette, Gérard Bois

\section{- To cite this version:}

Qiaorui Si, Patrick Dupont, Annie-Claude Bayeul-Lainé, Antoine Dazin, Olivier Roussette, et al.. Static pressure recovery analysis in the vane island diffuser of a centrifugal pump. Journal of Mechanical Science and Technology, 2016, 30 (2), pp.549-556. 10.1007/s12206-016-0108-7 . hal-02160463

\section{HAL Id: hal-02160463 \\ https://hal.science/hal-02160463}

Submitted on 19 Jun 2019

HAL is a multi-disciplinary open access archive for the deposit and dissemination of scientific research documents, whether they are published or not. The documents may come from teaching and research institutions in France or abroad, or from public or private research centers.
L'archive ouverte pluridisciplinaire HAL, est destinée au dépôt et à la diffusion de documents scientifiques de niveau recherche, publiés ou non, émanant des établissements d'enseignement et de recherche français ou étrangers, des laboratoires publics ou privés. 


\title{
Static pressure recovery analysis in the vane island diffuser of a centrifugal pump ${ }^{\dagger}$
}

\author{
Qiaorui $\mathrm{Si}^{1}$, Patrick Dupont ${ }^{2}$, Annie-Claude Bayeul-Lainé ${ }^{3}$, Antoine Dazin $^{3}$, \\ Olivier Roussette ${ }^{3}$ and Gérard Bois ${ }^{3, *}$ \\ ${ }^{1}$ National Research Center of Pumps, Jiangsu University, Zhenjiang 212013, China \\ ${ }^{2}$ LML, UMR CNRS 8107 Ecole Centrale de Lille, Lille 59046, France \\ ${ }^{3}$ LML, UMR CNRS 8107, Arts et Métiers PARISTECH, Lille 59046, France
}

\begin{abstract}
The overall performance of a vane-island type diffuser of a centrifugal pump model was obtained by means of directional probe traverses. These measurements were performed in an air model of a real hydraulic pump for five volume flow rates. Directional probe traverses are performed with a classical three-hole probe to cover most of the complete inlet section of the diffuser from hub to shroud and from pressure to suction side. Existing Particle image velocimetry (PIV) measurement results are also used to compare probe measurement results between the inlet and outlet throats of vane island diffuser at mid-span. Some assistance from already existing unsteady calculation, including leakage effects, is used to evaluate the numerical approach capability and to correctly define the mean initial conditions at impeller's outlet section. Pressure recovery and the measured total pressure loss levels inside this particular vane diffuser geometry are then calculated. Detailed analysis of the flow structure at the inlet section of the vane island diffuser is presented to focus on pressure evolution inside the entire diffuser section for different flow rates. The combined effects of incidence angle and blockage distributions along hub to shroud direction are found to play an important role on loss distribution in such a diffuser.
\end{abstract}

Keywords: Centrifugal pumps; Pressure recovery; Three-hole probe; PIV

\section{Introduction}

Pumps are essential energy conversion devices and fluidtransporting equipment. Therefore, a solid knowledge of the flow field within the impeller and diffuser of pumps is needed to obtain improved performance. Flow in radial pumps is highly three-dimensional, spatially non-uniform, intrinsically unsteady, and departs from axisymmetric or periodic assumptions, which were strongly highlighted by previous works [14]. Moreover, due to the rotor stator interaction effect, the diffuser vanes play a major role in establishing circumferential flow fields near the exit of the impeller, as shown by several studies [5-8]. However, unsteady effect may fundamentally change the loss inside the diffuser is still uncertain. Therefore, analyzing pressure recovery downstream the impeller is significant.

Numerical and experimental methods are two main ways to investigate the above aspects. However, the capability of Computational fluid dynamics (CFD) to precisely describe all local complex flow phenomena is limited. To validate the
CFD results, experiments are still needed to provide benchmark data. Simultaneously, numerical results promote analysis of experimental results [9]. Extensive experimental and numerical work has been conducted on a special pump model that works with air at the Laboratoire de Mécanique de Lille (LML, France). Several papers have also been published [1014]. The frozen rotor technique has been proven to be able to capture parts of the real flow characteristics of the impeller itself and cannot be used to obtain overall pump performances near design points even with reliable mesh configurations. However, for a wide range of mass flow rates, Cavazzini et al. [15] showed good comparisons between PIV measurement and full Unsteady Reynolds average Navier Stokes (URANS) calculations on the present pump model, taking into account the impeller blade's relative location to the vane diffuser channel. All these previous studies focus on the unsteady velocity fields only. To analyze the pressure recovery of the diffuser, pressure measurements must be processed and impeller outlet conditions have to be known prior to diffuser analysis. Among the several experimental techniques that are generally used, only the intrusive pressure probe is able to provide a total pressure evaluation as well as correct impeller and pump loss evaluations.

In this paper, a classical three-hole probe is used to cover 
Table 1. Parameters of the tested pump configurations.

\begin{tabular}{c|c}
\hline Name & Value \\
\hline Impeller inlet radius & $R_{1}=0.1411 \mathrm{~m}$ \\
\hline Impeller outlet radius & $R_{2}=0.2566 \mathrm{~m}$ \\
\hline Number of impeller blades & $Z_{\mathrm{i}}=7$ \\
\hline Wide of impeller outlet & $b_{2}=0.0385 \mathrm{~m}$ \\
\hline Impeller design flow rate & $Q_{\mathrm{n}}=0.3365 \mathrm{~m} / \mathrm{s}$ \\
\hline Rotation speed & $N=1710 \mathrm{rpm}$ \\
\hline Impeller tip velocity & $u_{2}=45.95 \mathrm{~m} / \mathrm{s}$ \\
\hline Diffuser inlet radius & $R_{3}=0.2736 \mathrm{~m}$ \\
\hline Diffuser outlet radius & $R_{4}=0.3978 \mathrm{~m}$ \\
\hline Number of diffuser vanes & $Z_{\mathrm{d}}=8$ \\
\hline Height from hub to shroud & $b_{3}=0.04 \mathrm{~m}$ \\
\hline Diffuser design flow rate & $Q_{\mathrm{d}}=0.8 Q_{\mathrm{n}}$ \\
\hline Impeller-diffuser radial gap & $1 \mathrm{~mm}$ \\
\hline
\end{tabular}

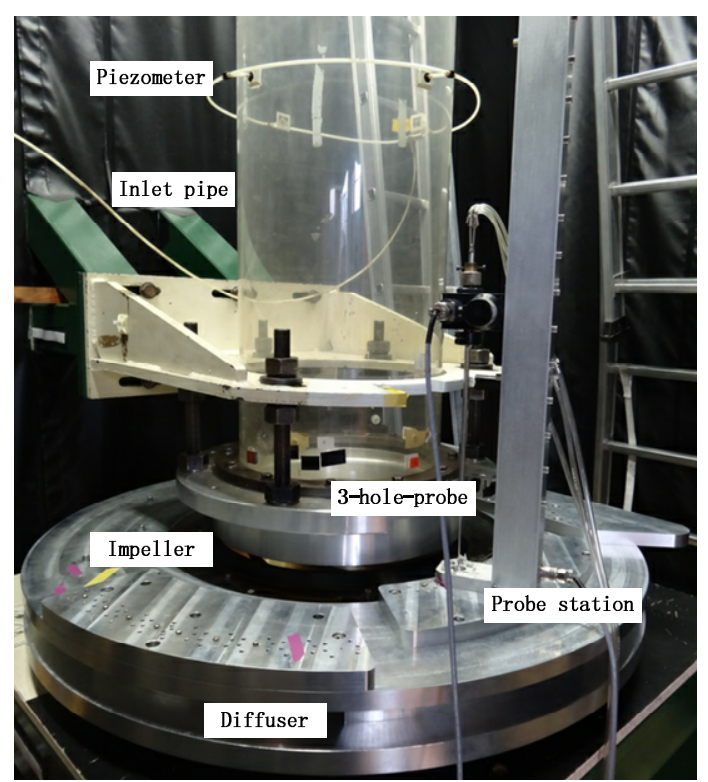

Fig. 1. Test rig.

most of the complete flow passage along the different sections of the diffuser. Mean velocity components obtained by the three hole probe are compared with instantaneous velocity fields from existing PIV results in order to show that a steady analysis can be performed inside the vane diffuser passage as far as overall performances are concerned.

\section{Experimental setup}

Tests have been performed in the air with the so-called "SHF" impeller on a test rig specially adapted to study impeller-diffuser interactions (Fig. 1). No volute was placed downstream of the vaned diffuser to avoid further interactions. Outlet pressure is at atmospheric conditions. Thus, the total pressure of the test rig is always below atmospheric pressure.

The main features of the components and specifications of

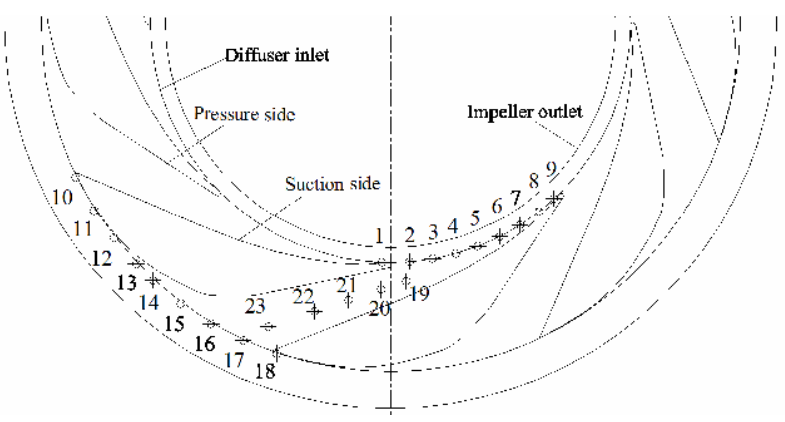

Fig. 2. Probe traverse locations in the diffuser.

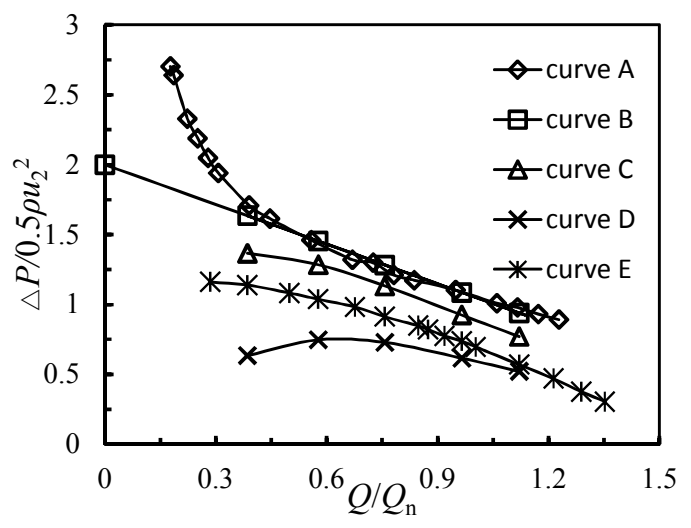

Fig. 3. Pressure coefficients versus flow rate.

the considered operating condition are summarized in Table 1. The diffuser design flow rate corresponds to $80 \%$ of the impeller design flow rate $\left(Q_{\mathrm{n}}=0.3365 \mathrm{~m}^{3} / \mathrm{s}\right)$. This configuration has been chosen to obtain a better pump performance at partial flow rate.

The experimental procedure that was chosen is used to perform hub to shroud traverses for several locations in between the pressure and suction side of one vane diffuser blade-toblade inlet section, and at several locations along the diffuser length, as shown in Fig. 2. The same three-hole directional probe proposed by Cherdieu [16] was used to perform these traverses by using the usual calibration procedure to obtain local mean radial and tangential velocity components as well as total and static pressure values. Probe traverses have been performed for five different operating points, namely, $Q / Q_{\mathrm{n}}=$ $0.386,0.581,0.762,0.968$ and 1.12 , with respect to nominal flow rate conditions of the impeller.

\section{Measurement results and discussion}

\subsection{Determination of impeller outlet overall mean conditions}

To determine real unsteady flow conditions at the impeller outlet section, measurements have to be performed somewhere close to the impeller exit. If the radial gap between the impeller exit and the vane diffuser inlet plane is close, then the measurements must be conducted close to the vane diffuser inlet plane. For the present experimental setup, a specific arrangement allows flow leakage between the fixed and rotating 
parts of the pump model just after the impeller outlet section. Due to low pressure, this leakage may enter the inlet section of the vane diffuser [17]. Thus, only the average impeller outlet performances were obtained by the following procedure, which would be considered the initial conditions for the vane diffuser performances.

The impeller mean overall performances curve is deduced from experimental results obtained by Barrand et al. [18] as shown in Fig. 3(curve A). They had been experimentally obtained by torque meters and validated in two different water test stands: at Institut National des Sciences Appliquées (INSA, France) and at École Polytechnique Fédérale de Lausanne (EPFL, Switzerland). The straight line (curve B) correctly fits this first curve with a wide range of flow rate (for $Q / Q_{\mathrm{n}}=0.5$ and above). It corresponds to the theoretical curve obtained by applying the Euler equation, with the assumption of no inlet swirl velocity conditions at the impeller inlet and a constant outlet relative angle $\beta_{2}$ equal to $14^{\circ}$ with the tangential direction.

The existing experimental impeller head curve allowed us to calculate the mean absolute tangential velocity. The mean radial velocity component is obtained according to the flow rate assuming constant velocity in the outlet plane of the impeller exit. Impeller efficiencies were obtained from existing 1D loss models, the results of which were similar to those obtained by using unsteady loss calculations from several CFD methods. The calculated real fluid total and static pressure coefficient at the impeller outlet are also given in Fig. 3, respectively on curves $\mathrm{C}$ and $\mathrm{D}$, for the five flow rates that were chosen for all measurements presented in this paper. These values were then taken as the initial conditions for all further performance calculations. The last curve shown in Fig. 3 (curve E) corresponds to the overall static pressure rise coefficient of the pump model.

\subsection{Vane diffuser inlet local conditions}

From the three-hole probe traverses, values of absolute velocity $v$ and absolute angle as well as total and static pressure, were obtained for each flow rate. An example is given in Fig. 4 at $Q / Q_{\mathrm{n}}=0.762$, which is close to the design diffuser flow rate.

Notably, the measurements were difficult to perform near the pressure and the suction wall side (positions 1 and 9 in Fig. 2) as a result of the blockage effect of the probe near the hub and shroud position because of its own dimensions. Therefore, almost 90 percent of the diffuser inlet section was explored. The main flow characteristics could be obtained after weighted average. Non-dimensional velocity $v^{*}$, nondimensional total pressure $P_{\text {tot }}{ }^{*}$, and non-dimensional static pressure $P_{\text {sta }}^{*}$ are defined as follows.

$$
\begin{aligned}
& v^{*}=v / u_{2} \\
& P_{\text {tot }}^{*}=\frac{P_{\text {tot }}-P_{\text {aspA-tot }}}{0.5 \rho u_{2}^{2}}
\end{aligned}
$$

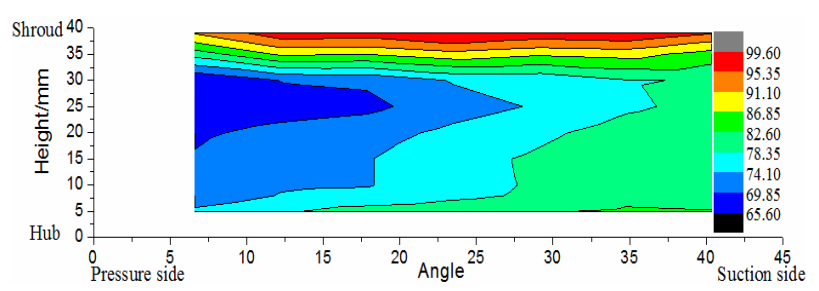

(a)

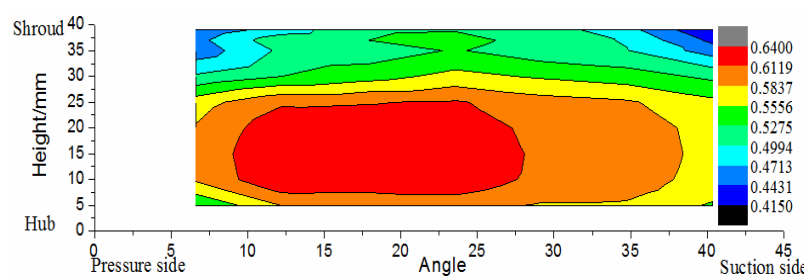

(b)

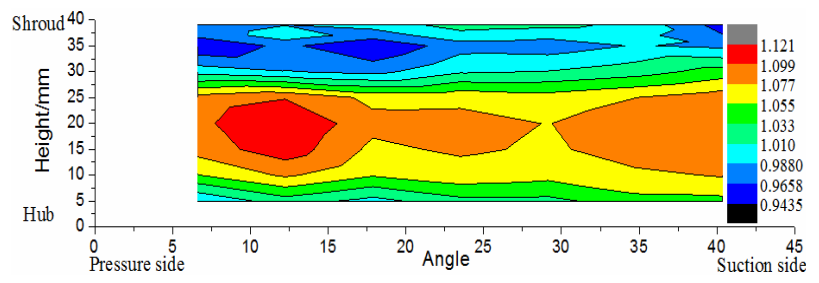

(c)

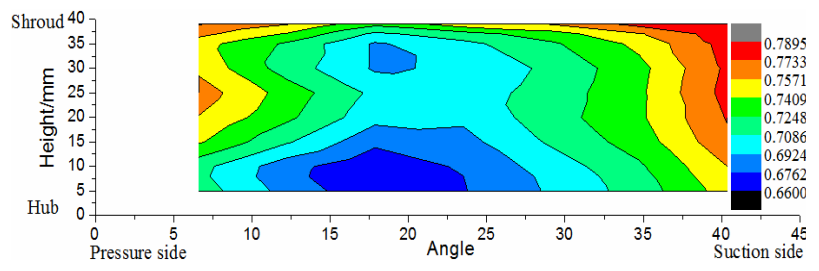

(d)

Fig. 4. Measurement results at $Q / Q_{\mathrm{n}}=0.762$ : (a) absolute angle; (b) non-dimensional absolute velocity; (c) non-dimensional total pressure field; (d) non-dimensional static pressure field.

$$
P_{\text {sta }}^{*}=\frac{P_{\text {sta }}-P_{\text {aspA }}}{0.5 \rho u_{2}^{2}}
$$

where $u_{2}$ stands for the impeller tip velocity, $P_{\text {aspA-tot }}$ means the total pressure and $P_{\text {aspa }}$ means the static pressure at the inlet pipe.

Fig. 4(a) shows that reverse flow is detected near the shroud for all blade-to-blade tangential positions that correspond to absolute angle values of more than $90^{\circ}$. This reverse flow region corresponds to 20 percent of the diffuser width and is associated with low absolute mean velocities, as shown in Fig. 4(b). The non-dimensional total and static pressure distributions given in Figs. 4(c) and (d), respectively, show that the maximum total pressure levels were located at the mid-span diffuser width and in the middle part of the blade-to-blade section toward the pressure side. The static pressure map indicates that low values are concentrated in the middle of the passage with an almost equivalent pressure level close to the pressure and suction side. This finding is consistent with the 


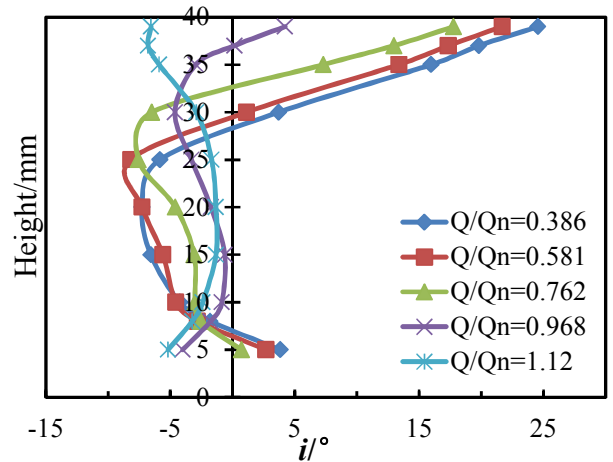

(a)

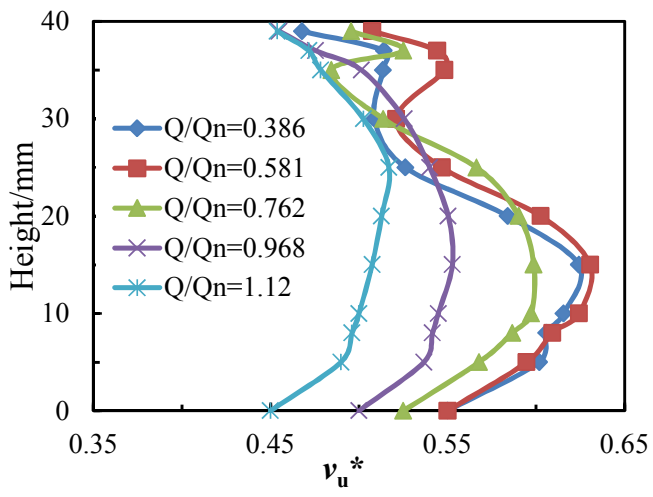

(c)

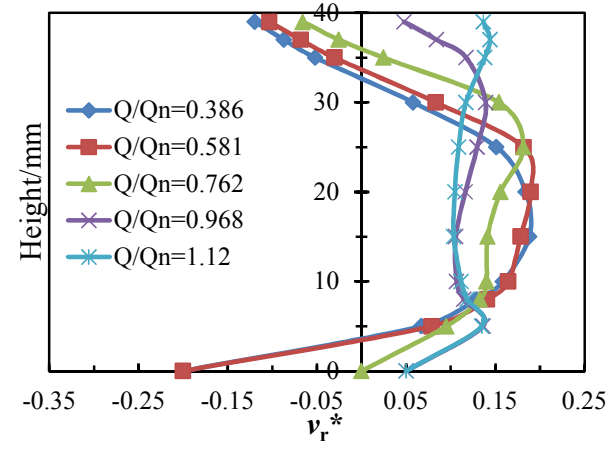

(b)

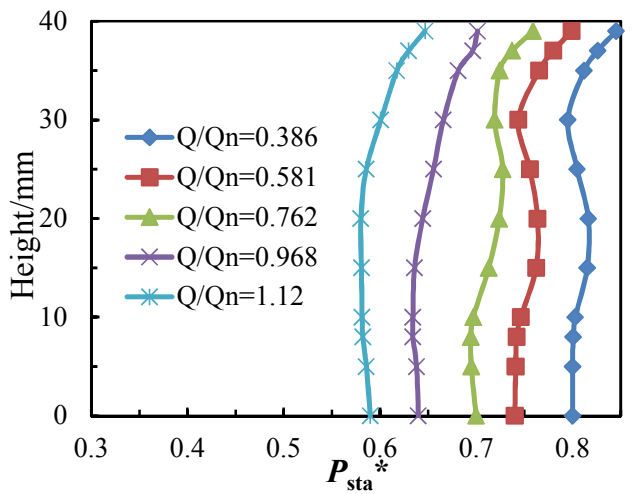

(d)

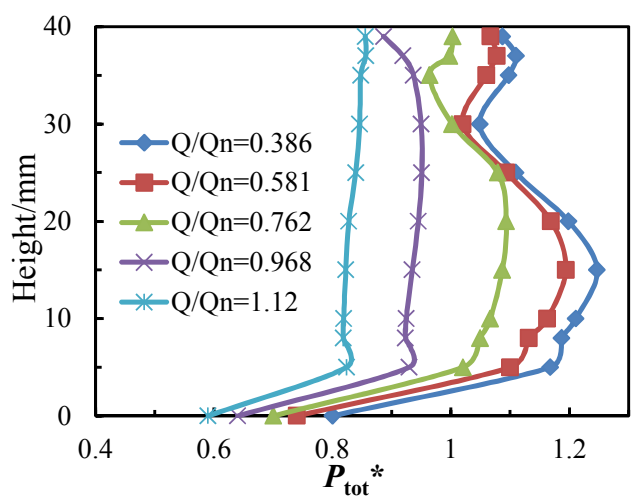

(e)

Fig. 5. Mean values at diffuser inlet: (a) mean incidence angle; (b) non-dimensional radial velocity; (c) non-dimensional tangential velocity; (d) nondimensional static pressure field; (e) non-dimensional total pressure.

fact that flow conditions are close to the design point of the vane diffuser.

The maximum total pressure coefficient value obtained by the probe measurements exactly corresponds to the mean total pressure value for $Q / Q_{\mathrm{n}}=0.762$ calculated at the impeller outlet section given in Fig. 3. This finding means that after mixing, a blade-to-blade loss distribution is obtained in the vaneless diffuser part considering downstream vane diffuser interactions. The corresponding maximum total pressure zone is then equivalent to isentropic flow conditions inside the vaneless diffuser part coming from the impeller outlet condition. This result also corresponds to a time averaged total pressure distribution because the combination of rotor-stator interactions and jet-wake structure that issued from the impeller itself leads to unsteady flow in this region.

\subsection{Vane diffuser hub to shroud distributions at inlet section}

Mass averaged value distributions can be obtained from previous maps. For the five measuring flow rates, the results for the incidence angle at the leading edge of the vane diffuser, non-dimensional radial and tangential velocity components, and non-dimensional static and total pressure are shown in Figs. 5(a)-(e), respectively. The incidence angle, which was 
obtained from the difference between the absolute flow angle $\alpha$ and the blade angle $\left(79.8^{\circ}\right)$, exhibits negative incidence in the middle of the hub to shroud section, as shown in Fig. 5(a). For low flow rates, the negative incidence values are pronounced because of strong blockage effects near the shroud with quite high positive incidence values. Small incidence values could be seen for the two largest flow rates because the radial velocity distribution (see Fig. 5(b)) is almost constant over the hub to shroud section. These aspects are considered for the analysis of the pressure recovery in the vane diffuser presented in Sec. 3.4.

For the three complementary flow rates, reverse flows occur near the shroud. In Fig. 5(c), the tangential velocity distribution is increasing with decreasing flow rate values, which is consistent with the increasing torque value initially seen on the theoretical head curve of Fig. 3. Static pressure distribution along the diffuser height (Fig. 5(d)) is almost constant. Local pressure gradients near the shroud indicate that some curvatures of the meridional streamlines may be present. The total pressure distributions in Fig. 5(e) are also consistent with the tangential velocity ones. Note that total pressure values given at the hub are not measured but obtained from the extrapolated value of static pressure at the hub in Fig. 5(d).

\subsection{Diffuser static pressure recovery}

Measured pressure recovery coefficient evolutions along the diffuser radius are given in Fig. 6(a). Initial pressure and velocity values are taken from impeller outlet values as stated in Sec. 3.1.

In the vaneless diffuser part, static pressure slightly increases for the low flow rate $Q / Q_{\mathrm{n}}=0.386$ and $Q / Q_{\mathrm{n}}=0.583$. This result is linked to sudden deceleration of the diffuser inlet flow conditions shown in Fig. 5. For all other flow rate values, static pressure remains constant in this diffuser part. For $Q / Q_{\mathrm{n}}=$ 0.762 , the static pressure value remains constant near the vane diffuser inlet throat that corresponds to positions 19 and 20. For $Q / Q_{\mathrm{n}}=0.386$ and 0.581 , the static pressure always increases rapidly before position 20 then rises gently. For the maximum flow rate value $Q / Q_{\mathrm{n}}=1.12$, no pressure recovery occurs mainly because of negative incidence on the leading edge of the vane. This negative incidence is associated with strong local flow blockage, which leads to strong acceleration in the vane diffuser channel associated with pressure losses. In Fig. $6(\mathrm{a})$, the theoretical pressure recovery factor, which was determined by using Eq. (4), was plotted based on the vane diffuser geometrical section evolution along the radius. In the following equation, $v_{\mathrm{R}}$ denotes the absolute velocity at radius $R$.

$$
\frac{P_{\mathrm{t}}}{0.5 \rho v_{2}^{2}}=1-\frac{v_{R}^{2}}{v_{2}^{2}} .
$$

The difference between theoretical and real measured final values from inlet to outlet diffuser sections is plotted in Fig. 6(b). The negative values at flow rates 0.968 and 1.12 might

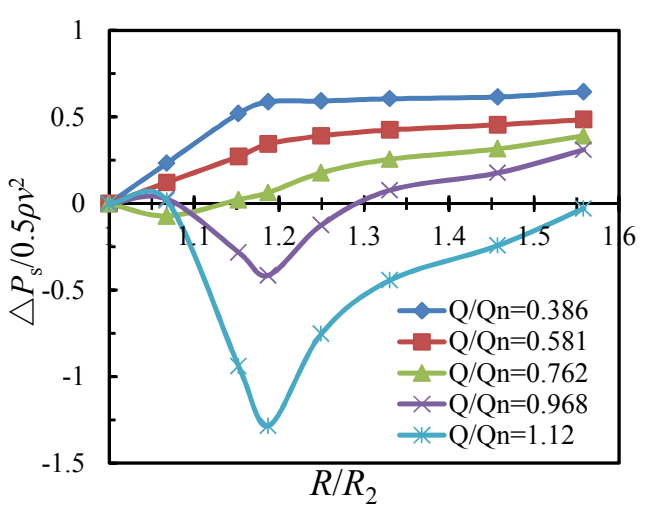

(a)

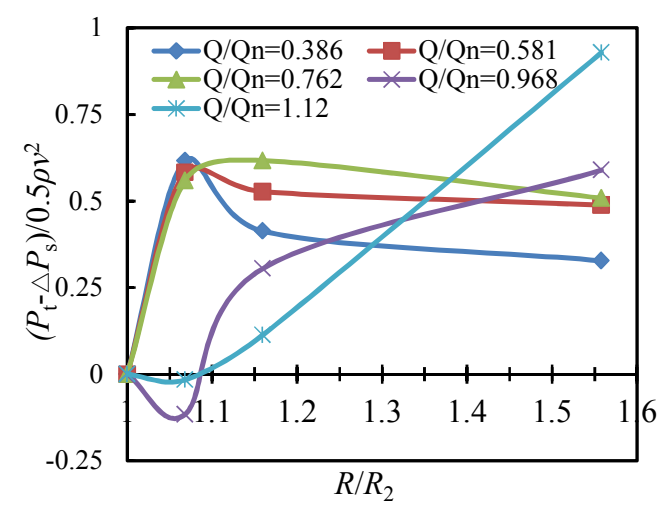

(b)

Fig. 6. Evolution of static pressure coefficients downstream the impeller: (a) theoretical and test pressure recovery results; (b) difference between theoretical and measured pressure recovery coefficients.

be caused by the entered leakage flow, which added energy to the diffuser part. These differences also related to the total loss coefficient. Close to the diffuser design point, pressure recovery factor is rather good close to reduced flow rates $Q / Q_{\mathrm{n}}=$ 0.762 and 0.581 . The minimum value, however, is reached for a lower reduced flow rate $Q / Q_{\mathrm{n}}=0.386$. At this flow rate, one can see that the local pressure recovery has already got a quite good value just after the first diffuser throat. More detailed analysis must be performed in order to explain such pressure recovery distributions.

\subsection{Comparison with PIV measurement}

The 2C-2D (two-dimensional with two cameras) high speed stereoscopic PIV measurements inside the diffuser channel were processed. Details on PIV techniques used, experimental setup, and result interpretations can be found in the Ref. [19]. The experimental procedure allowed the determination of the mean absolute velocities for up to 7 impeller angular positions relative to diffuser vanes, as shown in Fig. 7(a). Moreover, the time average values were plotted in Fig. 7 for different locations inside the diffuser channel along the radius that corresponds to probe measurement locations 19 to 23 (see Fig. 3). This method calculates the averaged radial and tangential 


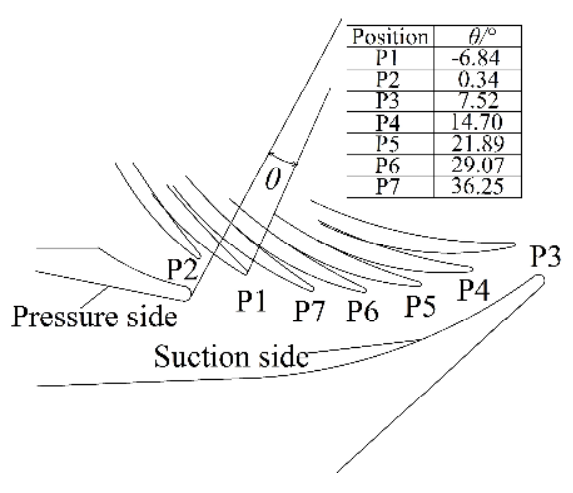

(a)

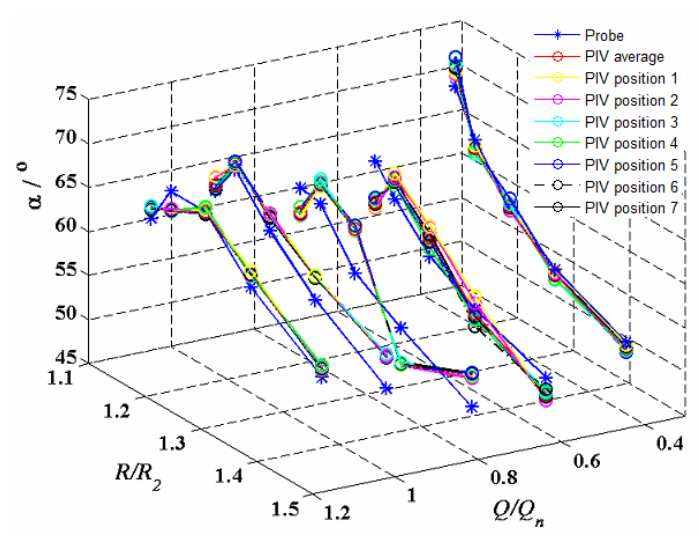

(b)

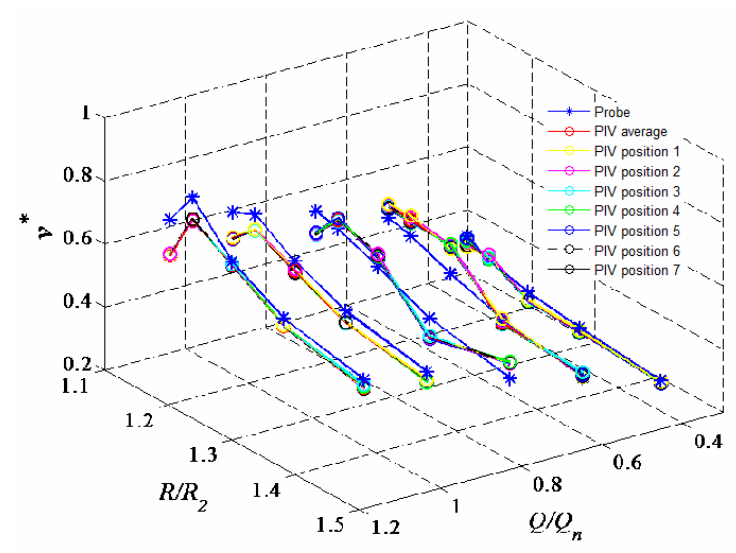

(c)

Fig. 7. Comparison between probe and PIV measurements: (a) scheme of the impeller positions; (b) flow angle comparison; (c) nondimensional absolute velocity comparison.

velocities first followed Eqs. (5) and (6), and then computes the flow angle and absolute velocity, as shown in Fig. 7. This paper presents only the comparison at mid-span due to space constraints.

$$
\overline{v_{r}}=\frac{\int_{\theta_{1}}^{\theta_{7}} v_{r}(\theta) d \theta}{\int_{\theta_{1}}^{\theta_{7}} \theta d \theta}
$$

$$
\overline{v_{u}}=\frac{\int_{\theta_{1}}^{\theta_{7}} v_{u}(\theta) \cdot v_{r}(\theta) d \theta}{\int_{\theta_{1}}^{\theta_{7}} v_{r}(\theta) d \theta} .
$$

Based on only the PIV results, the mean absolute angle and velocity distribution along the diffuser channel is constant no matter the impeller blade position and flow rate values. This result is due to the fact that unsteady effects from the impeller relative position with the vane diffuser channel almost vanish in the main flow considering time average values of velocity components that are going to be compared with the probe measurements. However, it does not mean that this unsteady flows character has no action on the turbulence itself. Probe measurement results agree with PIV ones, mainly downstream from point 21. At the front of the diffuser channel, probe measurements do not correctly fit the PIV ones. This incorrect fit may be due to the possibility that flow structure may be more tridimensional inside the diffuser channel according to strong incidence angle gradients along the diffuser width (as seen in Fig. 6(a)), which may create high axial velocity gradients between the pressure and the suction sides of the diffuser channel; these gradients cannot be measured by the probe itself. At high flow rates above the vane diffuser design flow rate, probe measurement values are always a little bit lower than the PIV values, which might be caused by the flow separation area and unsteady effects.

\section{Conclusions}

Detailed mean flow characteristics have been measured at five flow rates to analyze the mean inlet flow conditions of a vane diffuser of a laboratory centrifugal pump model that works with air. The initial conditions outside the impeller were used to process mean flow characteristics analysis. Local probe measurements inside the vane diffuser were also compared with PIV measurements. It can be concluded that:

(1) The flow could be considered as a steady one no matter the relative position of the impeller is as long as overall diffuser performances are concerned. This allows an evaluation of the pressure recovery of the vane diffuser for five different mass flow rates.

(2) Static pressure evolutions seem to be better for low flow rates. However, care must be taken on the local absolute initial velocity gradients from hub to shroud to ensure a complete analysis of the flow inside the vane diffuser itself.

(3) The pressure recovery coefficients of the vane diffuser were compared with two dimensional theoretical ones for all flow rates. The difference between theoretical and real values indicates that the best pressure recovery factor is obtained for the lowest flow rate $Q / Q_{\mathrm{n}}=0.386$. Deeper analysis must be performed in order to explain these performances. 


\section{Acknowledgment}

The researchers wish to thank French National Research Council CNRS for their financial supports. The authors also gratefully acknowledge the financial support by National Natural Science Foundation of China (Grant No. 51509108, 51409125), Natural Science Foundation of Jiangsu Province (Grant No. SBK2015042921, BK20140554), the Open Research Subject of Key Laboratory (Xihua University) of Fluid and Power Machinery (Grant No. szjj2015-016) and Senior Talent Foundation of Jiangsu University (15JDG048).

\section{Nomenclature}

$\theta \quad:$ Phase angle of the impeller blade

$\rho \quad:$ Density

$Q \quad$ : Volume flow rate

$v \quad:$ Absolute velocity

$v^{*} \quad$ : Non-dimensional absolute velocity

$P_{\text {tot }}{ }^{*} \quad$ : Non-dimensional total pressure

$P_{\text {sta }}{ }^{*} \quad$ : Non-dimensional static pressure

$v_{\mathrm{r}} \quad:$ Radial velocity

$v_{\mathrm{u}} \quad:$ Tangential velocity

$u_{2} \quad$ : Circumferential velocity at impeller tip

$P_{\text {sta }} \quad:$ Static pressure

$P_{\text {tot }} \quad:$ Total pressure

$P_{\text {aspA }}:$ Static pressure at inlet pipe

$P_{\text {aspA-tot }}$ : Total pressure at inlet pipe

$P_{\mathrm{a}} \quad:$ Theoretical pressure recovery factor

$\alpha \quad$ : Absolute flow angle measured from radial direction

$\beta \quad$ : Relative flow angle measured from radial direction

$i \quad$ : Incidence angle

$\triangle P \quad$ : Pressure difference

\section{References}

[1] M. Sinha and J. Katz, Quantitative visualization of the flow in a centrifugal pump with diffuser vanes. part I: on flow structures and turbulence, J. Fluids Engineering, 122 (2000) 97-107.

[2] S. Guo and Y. Maruta, Experimental investigations on pressure fluctuations and vibration of the impeller in a centrifugal pump with vaned diffusers, JSME International Journal, 48 (1) (2005) 136-143.

[3] K. Majidi, Numerical study of unsteady flow in a centrifugal pump, Journal of Turbomachinery, 127 (2005) 363-371.

[4] K. M. Guleren and A. Pinarbasi, Numerical simulation of the stalled flow within a vaned centrifugal pump, Proceedings of the Institution of Mechanical Engineers, Part C: Journal of Mechanical Engineering Science, 218 (2004) 425-435.

[5] S.-S. Hong and S.-H. Kang, Flow at the centrifugal pump impeller exit with the circumferential distortion of the outlet static pressure, Journal of Fluids Engineering, 126 (1) (2004) 81-86.
[6] G. Pavesi, G. Cavazzini and G. Ardizzon, Time-frequency characterization of the unsteady phenomena in a centrifugal pump, International Journal of Heat and Fluid Flow, 29 (2008) 1527-1540.

[7] J. Feng et al., Unsteady flow investigation in rotor-stator interface of a radial diffuser pump, Forsch Ingenieurwes, 74 (2010) 233-242.

[8] G. Cavazzini et al., Analysis of unsteady flow velocity fields inside the impeller of a radial flow pump: PIV measurements and numerical calculation comparisons, Proceedings of ASME-JSME-KSME Joint Fluids Engineering Conference, Hamamatsu, Shizuoka, Japan (2011).

[9] G. Cavazzini, G. Pavesi and G. Ardizzon, Pressure instabilities in a vaned centrifugal pump, Proceedings of the Institution of Mechanical Engineers, Part A, Journal of Power and Energy, 225 (7) (2011) 930-939.

[10] G. Wuibaut et al., Optical PIV and LDV comparisons of internal flow investigations in SHF impeller, Int. J. of Rotating Machinery (2006) 1-9.

[11] G. Wuibaut, P. Dupont, G. Caignaert and G. Bois, Rotor stator interactions in a vaned diffuser radial flow pump, Proceedings of the 22nd IAHR Symposium on Hydraulic Machinery and Systems, Stockholm, Sweden (2004).

[12] G. Wuibaut et al., PIV measurements at the outlet part of a radial flow pump impeller and the vaneless diffuser in design and off design conditions, 4th International Symposium on Particle Image Velocimetry, Göttingen, Germany (2001).

[13] A. Atif, S. Benmandsour, G. Bois and P. Dupont, Numerical and experimental comparison of the vaned diffuser interaction inside the impeller velocity field of a centrifugal impeller, Science China Technological Sciences, 54 (2011) 286-294.

[14] G. Cavazzini et al., Analysis of the rotor-stator interaction in a radial flow pump, La Houille Blanche (2009) 141-151.

[15] G. Cavazzini et al., Unsteady velocity PIV measurements and 3D numerical calculation comparisons inside the impeller of a radial pump model, 10th European Conference on Turbomachinery, Lappenranta, Finland (2013) 15-19.

[16] P. Cherdieu et al., Data reduction problems using a 3 holes directional pressure probe to investigate mean flow characteristics in the vaneless gap between impeller and diffuser radial pump, Proceedings of the 6th International Conference on Pumps and Fans with Compressors and Wind Turbines, Beijing, China (2013).

[17] A. C. Bayeul-Lainé et al., Fluid leakage effect on analysis of a vaned diffuser of SHF, 15th International Symposium on Transport Phenomena and Dynamics of Rotating Machinery, Hawai, Honolulu, USA (2014).

[18] J. P. Barrand et al., Synthesis of the results of tests air and water aimed at critical recirculating flow rates detections on the inlet and outlet of a centrifugal impeller, La Houille Blanche, 5 (1985).

[19] A. Dazin et al., High-speed stereoscopic PIV study of rotating instabilities in a radial vaneless diffuser, Exp. Fluids, 51 (2011) 83-93. 